\title{
$(H, \phi)-\eta$-Accretive Mappings and a New System of Generalized Variational Inclusions with $(H, \phi)-\eta$-Accretive Mappings in Banach Spaces
}

\author{
Sayyedeh Zahra Nazemi \\ Department of Mathematics, Yasouj University, Yasouj 75914, Iran \\ Correspondence should be addressed to Sayyedeh Zahra Nazemi, szahranazemi@gmail.com
}

Received 5 June 2011; Accepted 29 June 2011

Academic Editors: Y.-K. Chang and Z. Hou

Copyright (C) 2011 Sayyedeh Zahra Nazemi. This is an open access article distributed under the Creative Commons Attribution License, which permits unrestricted use, distribution, and reproduction in any medium, provided the original work is properly cited.

We introduce a new class of generalized accretive mappings, named $(H, \phi)-\eta$-accretive mappings, in Banach spaces. We define a resolvent operator associated with $(H, \phi)$ - $\eta$-accretive mappings and show its Lipschitz continuity. We also introduce and study a new system of generalized variational inclusions with $(H, \phi)-\eta$-accretive mappings in Banach spaces. By using the resolvent operator technique associated with $(H, \phi)-\eta$-accretive mappings, we construct a new iterative algorithm for solving this system of generalized variational inclusions in Banach spaces. We also prove the existence of solutions for the generalized variational inclusions and the convergence of iterative sequences generated by algorithm. Our results improve and generalize many known corresponding results.

\section{Introduction}

Variational inequalities and variational inclusions are among the most interesting and important mathematical problems and have been studied intensively in the past years since they have wide applications in mechanics, physics, optimization and control, nonlinear programming, economics and transportation equilibrium, and engineering sciences, and so forth (see, e.g., $[1-4])$.

Recently Ding [5], Huang and Fang [6], Verma [7], Fang and Huang [8, 9], Huang and Fang [10], Fang et al. [11], Kazmi and Khan [12], and Lan et al. [13, 14] introduced the concepts of $\eta$-subdifferential operators, maximal $\eta$-monotone operators, $A$-monotone operators, and $(H, \eta)$-monotone operators in Hilbert spaces, $H$-accretive operators, generalized $m$-accretive mappings, $(H, \eta)$-accretive operators, $P$ - $\eta$-accretive operators, and $(A, \eta)$ accretive mappings in Banach spaces, and their resolvent operators, respectively. In [15], Luo 
and Huang introduced a new concept of $(H, \phi)-\eta$-monotone mappings in Banach spaces and defined the proximal mapping associated with $(H, \phi)-\eta$-monotone mappings.

Motivated and inspired by the research work going on this field, in this paper, we introduce a new concept of $(H, \phi)-\eta$-accretive mappings and give the definition of its resolvent operator in Banach spaces. We also introduce and study a new system of generalized variational inclusions with $(H, \phi)$ - $\eta$-accretive mappings in Banach spaces, and we construct a new iterative algorithm for solving this system of generalized variational inclusions in Banach spaces. We also prove the existence of solutions for the generalized variational inclusions and the convergence of iterative sequences generated by algorithm. The results in this paper improve and extend some known results in the literature.

\section{Preliminaries}

Let $E$ be a real Banach space equipped with norm $\|\cdot\|$; let $E^{*}$ be the topological dual space of $E$; let $\langle\cdot, \cdot\rangle$ be the pair between $E$ and $E^{*}$; let $2^{E}$ be the power set of $E$; let $\widetilde{H}(\cdot, \cdot)$ be the Hausdorff metric on $\mathrm{CB}(E)$ defined by

$$
\widetilde{H}(A, B)=\max \left\{\sup _{x \in A} d(x, B), \sup _{y \in B} d(A, y)\right\}, \quad \forall A, B \in \mathrm{CB}(E) .
$$

Definition 2.1 (see $[16,17]$ ). For $q>1$, a mapping $J_{q}: E \rightarrow 2^{E^{*}}$ is said to be generalized duality mapping if it is defined by

$$
J_{q}(x)=\left\{f \in E^{*}:\langle x, f\rangle=\|x\|^{q},\|x\|^{q-1}=\|f\|\right\}, \quad \forall x \in E .
$$

In particular, $J_{2}$ is the usual normalized duality mapping on $E$.

It is well known that

$$
J_{q}(x)=\|x\|^{q-1} J_{2}(x), \quad \forall x(\neq 0) \in E .
$$

Note that if $E=\mathscr{\ell}$ is a real Hilbert space, then $J_{2}$ becomes the identity mapping on $\mathscr{\ell}$.

Definition 2.2 (see [18]). A Banach space $E$ is called smooth, if for every $x \in E$ with $\|x\|=1$, there exists a unique $f \in E^{*}$ such that $\|f\|=f(x)=1$. The modulus of $E$ is the function $\rho_{E}:[0, \infty) \rightarrow[0, \infty)$, defined by

$$
\rho_{E}(\tau)=\sup \left\{\frac{\|x+y\|+\|x-y\|}{2}-1: x, y \in E,\|x\|=1,\|y\|=\tau\right\} .
$$

Definition 2.3 (see [17]). The Banach space $E$ is said to be

(i) uniformly smooth, if

$$
\lim _{\tau \rightarrow 0} \frac{\rho_{E}(\tau)}{\tau}=0
$$


(ii) $q$-uniformly smooth, for $q>1$, if there exists a constant $\tau>0$ such that

$$
\rho_{E}(\tau) \leq c \tau^{q}, \quad \tau \in[0, \infty) .
$$

It is well known (see [16]) that

$$
L_{q}\left(\text { or } l_{q}\right) \text { is }\left\{\begin{array}{l}
q \text {-uniformly smooth, } \text { if } 1<q \leq 2, \\
\text { 2-uniformly smooth, if } q \geq 2
\end{array}\right.
$$

Note that if $E$ is uniformly smooth, $J_{q}$ becomes single-valued, and $J_{q}$ is single-valued if $E^{*}$ is strictly convex. In the sequel, unless otherwise specified, we always suppose that $E$ is a real Banach space such that $J_{q}$ is single-valued.

Lemma 2.4 (see [19]). Let $q>1$ be a real number and let $E$ be a smooth Banach space. Then $E$ is $q$-uniformly smooth if and only if there exists a constant $c_{q}>0$ such that for every $x, y \in E$,

$$
\|x+y\|^{q} \leq\|x\|^{q}+q\left\langle y, J_{q}(x)\right\rangle+c_{q}\|y\|^{q}
$$

Definition 2.5 (see $[9,20]$ ). Let $P: E \rightarrow E$ be a single-valued mapping. $P$ is said to be

(i) accretive if

$$
\left\langle P(x)-P(y), J_{q}(x-y)\right\rangle \geq 0, \quad \text { iff } x, y \in E ;
$$

(ii) strictly accretive if $P$ is accretive and

$$
\left\langle P(x)-P(y), J_{q}(x-y)\right\rangle=0 \quad \text { if } x=y
$$

(iii) $r$-strongly accretive if there exists a constant $r>0$ such that

$$
\left\langle P(x)-P(y), J_{q}(x-y)\right\rangle \geq r\|x-y\|^{q}, \quad \forall x, y \in E ;
$$

(iv) $m$-relaxed accretive if there exists a constant $m>0$ such that

$$
\left\langle P(x)-P(y), J_{q}(x-y)\right\rangle \geq-m\|x-y\|^{q}, \quad \forall x, y \in E ;
$$

(v) $(\alpha, \xi)$-relaxed cocoercive if there exist constants $\alpha, \xi>0$ such that

$$
\left\langle P(x)-P(y), J_{q}(x-y)\right\rangle \geq-\alpha\|P(x)-P(y)\|^{q}+\xi\|x-y\|^{q}, \quad \forall x, y \in E ;
$$

(vi) $s$-Lipschitz continuous if there exists a constant $s>0$ such that

$$
\|P(x)-P(y)\| \leq s\|x-y\|, \quad \forall x, y \in E .
$$


Definition 2.6 (see [12]). A mapping $\eta: E \times E \rightarrow E$ is said to be $\tau$-Lipschitz continuous if there exists a constant $\tau>0$ such that

$$
\|\eta(x, y)\| \leq \tau\|x-y\|, \quad \forall x, y \in E .
$$

Definition 2.7 (see [20]). Let $\eta: E \times E \rightarrow E$ and $P: E \rightarrow E$ be single-valued mappings. Then a multi-valued mapping $T: E \rightarrow 2^{E}$ is said to be

(i) $\eta$-accretive if

$$
\left\langle u-v, J_{q}(\eta(x, y))\right\rangle \geq 0, \quad \forall x, y \in E, \forall u \in T(x), v \in T(y)
$$

(ii) strictly $\eta$-accretive if

$$
\left\langle u-v, J_{q}(\eta(x, y))\right\rangle \geq 0, \quad \forall x, y \in E, \forall u \in T(x), v \in T(y),
$$

and equality holds if and only if $x=y$;

(iii) $\gamma$-strongly $\eta$-accretive if there exists a constant $\gamma>0$ such that

$$
\left\langle u-v, J_{q}(\eta(x, y))\right\rangle \geq \gamma\|x-y\|^{q}, \quad \forall x, y \in E, \forall u \in T(x), v \in T(y) ;
$$

(iv) $m$-relaxed $\eta$-accretive if there exists a constant $m>0$ such that

$$
\left\langle u-v, J_{q}(\eta(x, y))\right\rangle \geq-m\|x-y\|^{q}, \quad \forall x, y \in E, \forall u \in T(x), v \in T(y) ;
$$

(v) $\eta$ - $m$-accretive if $T$ is $\eta$-accretive and $(I+\rho T)(E)=E$ holds for all $\rho>0$;

(vi) $P$ - $\eta$-accretive if $T$ is $\eta$-accretive and $(P+\rho T)(E)=E$ holds for all $\rho>0$.

Definition 2.8. Let $E_{1}, E_{2}, \ldots, E_{n}$ be real Banach spaces, and, for $i=1,2, \ldots, n$, let $N_{i}$ : $\prod_{j=1}^{n} E_{j} \rightarrow E_{i}$ be a single-valued mapping. Then $N_{i}$ is said to be $\xi_{i j}$-Lipschitz continuous in the $j$ th argument, if there exists a constant $\xi_{i j}>0$ such that

$$
\begin{gathered}
\left\|N_{i}\left(x_{1}, \ldots, x_{j-1}, y_{j 1}, x_{j+1}, \ldots, x_{n}\right)-N_{i}\left(x_{1}, \ldots, x_{j-1}, y_{j 2}, x_{j+1}, \ldots, x_{n}\right)\right\| \\
\leq \xi_{i j}\left\|y_{j 1}-y_{j 2}\right\|, \quad \forall y_{j 1}, y_{j 2} \in E_{j}, x_{i} \in E_{i}(i \in\{1,2, \ldots, n\}, i \neq j) .
\end{gathered}
$$

Definition 2.9. Let $A: E \rightarrow \mathrm{CB}(E)$ be a set-valued mapping. $A$ is said to be $\widetilde{H}$-Lipschitz continuous, if there exists a constant $t>0$ such that

$$
\widetilde{H}(A(x), B(y)) \leq t\|x-y\|, \quad \forall x, y \in E,
$$

where $\widetilde{H}(\cdot, \cdot)$ denotes the Hausdorff metric on $\mathrm{CB}(E)$. 
Definition 2.10. Let $\mathscr{\ell}$ be a Hilbert space, and let $H: \mathscr{l} \rightarrow \mathscr{H}$ be a single-valued mapping. $H$ is said to be

(i) coercive if

$$
\lim _{\|x\| \rightarrow \infty} \frac{\langle H(x), x\rangle}{\|x\|}=+\infty
$$

(ii) hemicontinuous if for any fixed $x, y, z \in \mathscr{d}$, the function $t \rightarrow\langle H(x+t y), z\rangle$ is continuous at $0^{+}$.

\section{3. $(H, \phi)$ - $\eta$-Accretive Mappings}

In this section, we will introduce a new class of generalized accretive mappings, $(H, \phi)-\eta$ accretive mappings, and discuss some properties of $(H, \phi)$ - $\eta$-accretive mappings.

Definition 3.1. Let $E$ be a Banach space and let $H, \phi: E \rightarrow E, \eta: E \times E \rightarrow E$ be singlevalued mappings and $M: E \rightarrow 2^{E}$ a multi-valued mapping. The mapping $M$ is said to be a $(H, \phi)$ - $\eta$-accretive mapping, if $\phi \circ M$ is $\eta$-accretive and $(H+\phi \circ M)(E)=E$.

Remark 3.2. (i) If $M$ is $\eta$-accretive and $\phi(x)=\lambda x$, for all $x \in E, \lambda>0$, then $(H, \phi)$ - $\eta$-accretive mapping reduces to the $P$ - $\eta$-accretive mapping studied by Kazmi and Khan [12].

(ii) If $\eta(x, y)=x-y$, for all $x, y \in E, M$ is $\eta$-accretive, and $\phi(x)=\lambda x$, for all $x \in E$, $\lambda>0$, then $(H, \phi)-\eta$-accretive mapping reduces to the $H$-accretive mapping studied by Fang and Huang [9].

(iii) If $M$ is $m$-relaxed $\eta$-accretive and $\phi(x)=\lambda x$, for all $x \in E, \lambda>0$, then $(H, \phi)-\eta$ accretive mapping reduces to the $(A, \eta)$-accretive mapping studied by Lan et al. [14].

Similarly, we give the following definition.

Definition 3.3. Let $H, \phi: E \rightarrow E$ be single-valued mappings and $M: E \rightarrow 2^{E}$ a multi-valued mapping. The mapping $M$ is said to be a $(H, \phi)$-accretive mapping, if $\phi \circ M$ is accretive and $(H+\phi \circ M)(E)=E$.

Example 3.4. Let $\mathscr{t}$ be a Hilbert space and for every $x, y \in \mathscr{l}, \eta(x, y)=x-y, \phi(x)=\lambda x$, where $\ell>0$ is a constant. Let $M: \mathscr{d} \rightarrow 2^{\mathscr{d}}$ be a maximal monotone mapping and $H: \mathscr{d} \rightarrow \mathscr{d}$ a bounded, coercive, hemicontinuous, and $\alpha$-strongly $\eta$-accretive mapping. Then it follows from Corollary 32.26 of [21] that $M$ is $(H, \phi)$ - $\eta$-accretive mapping.

Theorem 3.5. Let $\phi: E \rightarrow E, \eta: E \times E \rightarrow E$ be single-valued mappings, $H: E \rightarrow E$ a strictly $\eta$-accretive mapping, and $M: E \rightarrow 2^{E} a(H, \phi)$ - $\eta$-accretive mapping. Then $(H+\phi \circ M)^{-1}$ is a single-valued mapping.

Proof. For any given $z \in E$, let $x, y \in(H+\phi \circ M)^{-1}(z)$. It follows that

$$
z-H(x) \in \phi \circ M(x), \quad z-H(y) \in \phi \circ M(y) .
$$

Then $\eta$-accretivity of $\phi \circ M$ implies that

$$
\left\langle z-H(x)-(z-H(y)), J_{q}(\eta(x, y))\right\rangle=\left\langle-H(x)+H(y), J_{q}(\eta(x, y))\right\rangle \geq 0 .
$$


This implies that $x=y$ and so $(H+\phi \circ M)^{-1}$ is a single-valued mapping. This completes the proof.

By Theorem 3.5, we can define the resolvent operator $J_{M, \phi}^{H, \eta}$ associated with an $(H, \phi)-\eta$ accretive mapping $M$ as follows.

Definition 3.6. Let $\phi: E \rightarrow E, \eta: E \times E \rightarrow E$ be single-valued mappings, let $H: E \rightarrow E$ be a strictly $\eta$-accretive mapping, and let $M: E \rightarrow 2^{E}$ be a $(H, \phi)$ - $\eta$-accretive mapping. A resolvent operator $J_{M, \phi}^{H, \eta}: E \rightarrow E$ is defined by

$$
J_{M, \phi}^{H, \eta}(x)=(H+\phi \circ M)^{-1}(x), \quad \forall x \in E .
$$

Remark 3.7. (i) If $M$ is $\eta$-accretive and $\phi(x)=\lambda x$, for all $x \in E, \lambda>0$, then the resolvent operator $J_{M, \phi}^{H, \eta}$ reduces to the $P-\eta$-proximal point mapping $J_{\eta, \rho}^{M}$ introduced by Kazmi and Khan [12].

(ii) If $\eta(x, y)=x-y$, for all $x, y \in E, M$ is $\eta$-accretive, and $\phi(x)=\lambda x$, for all $x \in E$, $\lambda>0$, then the resolvent operator $J_{M, \phi}^{H, \eta}$ reduces to the proximal-point mapping introduced by Fang and Huang [9].

(iii) If $M$ is $m$-relaxed $\eta$-accretive and $\phi(x)=\lambda x$, for all $x \in E, \lambda>0$, then the resolvent operator $J_{M, \phi}^{H, \eta}$ reduces to the resolvent operator $R_{\eta, M}^{\rho, A}$ introduced by Lan at el. [14].

Theorem 3.8. Let $\phi: E \rightarrow E$ be a single-valued mapping, let $\eta: E \times E \rightarrow E$ be a $\tau$-Lipschitz continuous mapping, let $H: E \rightarrow E$ be a $\gamma$-strongly $\eta$-accretive mapping, and let $M: E \rightarrow 2^{E}$ be a $(H, \phi)$ - $\eta$-accretive mapping. Then the resolvent operator $J_{M, \phi}^{H, \eta}$ is Lipschitz continuous with constant $\tau^{q-1} / \gamma$, that is,

$$
\left\|J_{M, \phi}^{H, \eta}(x)-J_{M, \phi}^{H, \eta}(y)\right\| \leq \frac{\tau^{q-1}}{r}\|x-y\|, \quad \forall x, y \in E
$$

Proof. Let $x, y$ be any given points in $E$, it follows from Definition 3.6 that

$$
J_{M, \phi}^{H, \eta}(x)=(H+\phi \circ M)^{-1}(x), \quad J_{M, \phi}^{H, \eta}(y)=(H+\phi \circ M)^{-1}(y) .
$$

This implies that

$$
\begin{gathered}
x-H\left(J_{M, \phi}^{H, \eta}(x)\right) \in \phi \circ M\left(J_{M, \phi}^{H, \eta}(x)\right), \\
y-H\left(J_{M, \phi}^{H, \eta}(y)\right) \in \phi \circ M\left(J_{M, \phi}^{H, \eta}(y)\right) .
\end{gathered}
$$

Since $M$ is $(H, \phi)$ - $\eta$-accretive, we have

$$
\begin{aligned}
& \left\langle x-H\left(J_{M, \phi}^{H, \eta}(x)\right)-\left(y-H\left(J_{M, \phi}^{H, \eta}(y)\right)\right), J_{q}\left(\eta\left(J_{M, \phi}^{H, \eta}(x), J_{M, \phi}^{H, \eta}(y)\right)\right)\right\rangle= \\
& \left\langle x-y-\left(H\left(J_{M, \phi}^{H, \eta}(x)\right)-H\left(J_{M, \phi}^{H, \eta}(y)\right)\right), J_{q}\left(\eta\left(J_{M, \phi}^{H, \eta}(x), J_{M, \phi}^{H, \eta}(y)\right)\right)\right\rangle \geq 0 .
\end{aligned}
$$


The inequality above implies that

$$
\begin{aligned}
\|x-y\|\left\|\eta\left(J_{M, \phi}^{H, \eta}(x), J_{M, \phi}^{H, \eta}(y)\right)\right\|^{q-1} & =\|x-y\|\left\|J_{q}\left(\eta\left(J_{M, \phi}^{H, \eta}(x), J_{M, \phi}^{H, \eta}(y)\right)\right)\right\| \\
& \geq\left\langle x-y, J_{q}\left(\eta\left(J_{M, \phi}^{H, \eta}(x), J_{M, \phi}^{H, \eta}(y)\right)\right)\right\rangle \\
& \geq\left\langle H\left(J_{M, \phi}^{H, \eta}(x)\right)-H\left(J_{M, \phi}^{H, \eta}(y)\right), J_{q}\left(\eta\left(J_{M, \phi}^{H, \eta}(x), J_{M, \phi}^{H, \eta}(y)\right)\right)\right\rangle \\
& \geq \gamma\left\|J_{M, \phi}^{H, \eta}(x)-J_{M, \phi}^{H, \eta}(y)\right\|^{q} .
\end{aligned}
$$

Since $\eta$ is Lipschitz continuous with a constant $\tau$, we have

$$
\left\|\eta\left(J_{M, \phi}^{H, \eta}(x), J_{M, \phi}^{H, \eta}(y)\right)\right\| \leq \tau\left\|J_{M, \phi}^{H, \eta}(x)-J_{M, \phi}^{H, \eta}(y)\right\| .
$$

It follows from (3.8) and (3.9) that

$$
\|x-y\| \tau^{q-1}\left\|J_{M, \phi}^{H, \eta}(x)-J_{M, \phi}^{H, \eta}(y)\right\|^{q-1} \geq \gamma\left\|J_{M, \phi}^{H, \eta}(x)-J_{M, \phi}^{H, \eta}(y)\right\|^{q}
$$

Hence, we get

$$
\left\|J_{M, \phi}^{H, \eta}(x)-J_{M, \phi}^{H, \eta}(y)\right\| \leq \frac{\tau^{q-1}}{r}\|x-y\| .
$$

This completes the proof.

\section{A New System of Generalized Variational Inclusions}

In this section, we will introduce a new system of generalized variational inclusions with $(H, \phi)-\eta$-accretive mappings and construct a new iterative algorithm for solving this system of generalized variational inclusions. In what follows, for each $i=1,2,3$, suppose that $E_{i}$ is a Banach space, $H_{i}, f_{i}, \phi_{i}, p_{i}: E_{i} \rightarrow E_{i}, \eta_{i}: E_{i} \times E_{i} \rightarrow E_{i}, F_{i}, N_{i}: \prod_{k=1}^{3} E_{k} \rightarrow E_{i}$ are singlevalued mappings, $T_{k i}: E_{k} \rightarrow \mathrm{CB}\left(E_{i}\right)$ is a set-valued mapping, and $M_{i}: E_{i} \times E_{i} \rightarrow 2^{E_{i}}$ is a $\left(H_{i}, \phi_{i}\right)-\eta_{i}$-accretive mapping in the second argument. Assume that $f_{i}\left(E_{i}\right) \cap \operatorname{dom} M\left(w_{i}, \cdot\right) \neq \emptyset$, for each $w_{i} \in E_{\mathrm{i}}$. We consider the following system of generalized variational inclusions. Find $\left(x_{1}, x_{2}, x_{3}, u_{11}, u_{12}, u_{13}, u_{21}, u_{22}, u_{23}, u_{31}, u_{32}, u_{33}\right)$ such that for each $i=1,2,3, x_{i} \in E_{i}, u_{1 i} \in$ $T_{1 i}\left(x_{1}\right), u_{2 i} \in T_{2 i}\left(x_{2}\right), u_{3 i} \in T_{3 i}\left(x_{3}\right)$, and

$$
0 \in F_{i}\left(x_{1}-p_{1}\left(x_{1}\right), x_{2}-p_{2}\left(x_{2}\right), x_{3}-p_{3}\left(x_{3}\right)\right)+N_{i}\left(u_{i 1}, u_{i 2}, u_{i 3}\right)+M_{i}\left(x_{i}, f_{i}\left(x_{i}\right)\right) .
$$

The following are some special cases of problem (4.1).

(i) If $E_{1}=\mathscr{t}_{1}, E_{2}=\mathscr{t}_{2}$, and $E_{3}=\mathscr{H}_{3}$ are three Hilbert spaces, and, for each $i=1,2,3$, $p_{i}=0, N_{i}=0, M_{1}\left(x, f_{1}(x)\right)=\partial_{\eta_{1}} \varphi_{1}(x)$, for all $x \in E_{1}, M_{2}\left(y, f_{2}(y)\right)=\partial_{\eta_{2}} \varphi_{2}(y)$, for all $y \in E_{2}$, and $M_{3}\left(z, f_{3}(z)\right)=\partial_{\eta_{3}} \varphi_{3}(z)$, for all $z \in E_{3}$, where $\varphi_{i}: E_{i} \rightarrow R \cup\{\infty\}$ is a proper lower 
semicontinuous and $\eta_{i}$-subdifferential function, $\partial_{\eta_{1}} \varphi_{1}(x)$ is the $\eta_{1}$-subdifferential of $\varphi_{1}$ at $x$, $\partial_{\eta_{2}} \varphi_{2}(y)$ is the $\eta_{2}$-subdifferential of $\varphi_{2}$ at $y$, and $\partial_{\eta_{3}} \varphi_{3}(z)$ is the $\eta_{3}$-subdifferential of $\varphi_{3}$ at $z$, then SGVIP (4.1) reduces to the following system of variational inequalities, which is to find $\left(x_{1}, x_{2}, x_{3}\right) \in E_{1} \times E_{2} \times E_{3}$ such that

$$
\begin{aligned}
& \left\langle F_{1}\left(x_{1}, x_{2}, x_{3}\right), \eta_{1}\left(a, x_{1}\right)\right\rangle+\varphi_{1}(a)-\varphi_{1}\left(x_{1}\right) \geq 0, \quad \forall a \in E_{1}, \\
& \left\langle F_{2}\left(x_{1}, x_{2}, x_{3}\right), \eta_{2}\left(b, x_{2}\right)\right\rangle+\varphi_{2}(b)-\varphi_{2}\left(x_{2}\right) \geq 0, \quad \forall b \in E_{2}, \\
& \left\langle F_{3}\left(x_{1}, x_{2}, x_{3}\right), \eta_{3}\left(c, x_{3}\right)\right\rangle+\varphi_{3}(c)-\varphi_{3}\left(x_{3}\right) \geq 0, \quad \forall c \in E_{3} .
\end{aligned}
$$

If $\eta_{1}(a, x)=a-x$, for all $a, x \in E_{1}, \eta_{2}(b, y)=b-y$, for all $b, y \in E_{2}, \eta_{3}(c, z)=c-z$, for all $c, z \in E_{3}$, and $M_{1}\left(x, f_{1}(x)\right)=\partial \varphi_{1}(x)$ is the subdifferential of $\varphi_{1}$ at $x, M_{2}\left(y, f_{2}(y)\right)=\partial \varphi_{2}(y)$ is the subdifferential of $\varphi_{2}$ at $y$, and $M_{3}\left(z, f_{3}(z)\right)=\partial \varphi_{3}(z)$ is the subdifferential of $\varphi_{3}$ at $z$, then problem (4.2) reduces to the following system of variational inequalities, which is to find $\left(x_{1}, x_{2}, x_{3}\right) \in E_{1} \times E_{2} \times E_{3}$ such that

$$
\begin{aligned}
& \left\langle F_{1}\left(x_{1}, x_{2}, x_{3}\right), a-x_{1}\right\rangle+\varphi_{1}(a)-\varphi_{1}\left(x_{1}\right) \geq 0, \quad \forall a \in E_{1}, \\
& \left\langle F_{2}\left(x_{1}, x_{2}, x_{3}\right), b-x_{2}\right\rangle+\varphi_{2}(b)-\varphi_{2}\left(x_{2}\right) \geq 0, \quad \forall b \in E_{2}, \\
& \left\langle F_{3}\left(x_{1}, x_{2}, x_{3}\right), c-x_{3}\right\rangle+\varphi_{3}(c)-\varphi_{3}\left(x_{3}\right) \geq 0, \quad \forall c \in E_{3} .
\end{aligned}
$$

If $M_{1}\left(x, f_{1}(x)\right)=\partial \delta_{k_{1}}(x), M_{2}\left(y, f_{2}(y)\right)=\partial \delta_{k_{2}}(y)$, and $M_{3}\left(z, f_{3}(z)\right)=\partial \delta_{k_{3}}(z)$, for all $x \in K_{1}, y \in K_{2}$, and $z \in K_{3}$, where $K_{1} \subset E_{1}, K_{2} \subset E_{2}$, and $K_{3} \subset E_{3}$ are three nonempty, closed, and convex subsets, $\delta_{k_{1}}, \delta_{k_{2}}$, and $\delta_{k_{3}}$ denote the indicator functions of $K_{1}, K_{2}$, and $K_{3}$, respectively, then problem (4.3) reduces to the following system of variational inequalities, which is to find $\left(x_{1}, x_{2}, x_{3}\right) \in E_{1} \times E_{2} \times E_{3}$ such that

$$
\begin{array}{ll}
\left\langle F_{1}\left(x_{1}, x_{2}, x_{3}\right), a-x_{1}\right\rangle \geq 0, & \forall a \in K_{1}, \\
\left\langle F_{2}\left(x_{1}, x_{2}, x_{3}\right), b-x_{2}\right\rangle \geq 0, & \forall b \in K_{2}, \\
\left\langle F_{3}\left(x_{1}, x_{2}, x_{3}\right), c-x_{3}\right\rangle \geq 0, & \forall c \in K_{3} .
\end{array}
$$

If $E_{1}=E_{2}=E_{3}=\mathscr{H}$ is a Hilbert space, $K_{1}=K_{2}=K_{3}=K$ is a nonempty, closed, and convex subset, $F_{1}(x, y, z)=s T_{1}(y, z, x)+x-y, F_{2}(x, y, z)=t T_{2}(z, x, y)+y-z$, and $F_{3}(x, y, z)=r T_{3}(x, y, z)+z-x$, for all $x, y, z \in K$, where $T_{1}, T_{2}, T_{3}: K \times K \times K \rightarrow$ de are mappings on $K \times K \times K, r, s, t>0$ are three numbers, then problem (4.4) reduces to the following system of variational inequalities, which is to find $x_{1}, x_{2}, x_{3} \in K$ such that

$$
\begin{array}{ll}
\left\langle s T_{1}\left(x_{2}, x_{3}, x_{1}\right)+x_{1}-x_{2}, a-x_{1}\right\rangle \geq 0, & \forall a \in K, \\
\left\langle t T_{2}\left(x_{3}, x_{1}, x_{2}\right)+x_{2}-x_{3}, a-x_{2}\right\rangle \geq 0, & \forall a \in K, \\
\left\langle r T_{3}\left(x_{1}, x_{2}, x_{3}\right)+x_{3}-x_{1}, a-x_{3}\right\rangle \geq 0, & \forall a \in K .
\end{array}
$$

Problem (4.5) was introduced and studied by Cho and Qin [22]. 
(ii) If $E_{2}=E_{3}, F_{1}(\cdot, \cdot, \cdot)=F_{1}(\cdot, \cdot), F_{2}(\cdot, \cdot, \cdot)=F_{2}(\cdot, \cdot)$, and $F_{2}=F_{3}$ and for each $i=1,2,3$, $p_{i}=0, N_{i}=0, M_{2}\left(y, f_{2}(y)\right)=M_{3}\left(z, f_{3}(z)\right)$, for all $(y, z) \in E_{2} \times E_{3}$, then SGVIP (4.1) reduces to the following system of variational inclusions, which is to find $\left(x_{1}, x_{2}\right) \in E_{1} \times E_{2}$ such that

$$
\begin{aligned}
& 0 \in F_{1}\left(x_{1}, x_{2}\right)+M_{1}\left(x_{1}, f_{1}\left(x_{1}\right)\right), \\
& 0 \in F_{2}\left(x_{1}, x_{2}\right)+M_{2}\left(x_{2}, f_{2}\left(x_{2}\right)\right) .
\end{aligned}
$$

If $E_{1}=\mathscr{t}_{1}$ and $E_{2}=\mathscr{\ell}_{2}$ are two Hilbert spaces and $M_{1}\left(x, f_{1}(x)\right)=\partial_{\eta_{1}} \varphi_{1}(x)$, for all $x \in E_{1}, M_{2}\left(y, f_{2}(y)\right)=\partial_{\eta_{2}} \varphi_{2}(y)$, for all $y \in E_{2}$, then the problem (4.6) reduces to the following system of variational inequalities, which is to find $\left(x_{1}, x_{2}\right) \in E_{1} \times E_{2}$ such that

$$
\begin{array}{ll}
\left\langle F_{1}\left(x_{1}, x_{2}\right), \eta_{1}\left(a, x_{1}\right)\right\rangle+\varphi_{1}(a)-\varphi_{1}\left(x_{1}\right) \geq 0, & \forall a \in E_{1}, \\
\left\langle F_{2}\left(x_{1}, x_{2}\right), \eta_{2}\left(b, x_{2}\right)\right\rangle+\varphi_{2}(b)-\varphi_{2}\left(x_{2}\right) \geq 0, & \forall b \in E_{2} .
\end{array}
$$

If $\eta_{1}(a, x)=a-x$, for all $a, x \in E_{1}, \eta_{2}(b, y)=b-y$, for all $b, y \in E_{2}, M_{1}\left(x, f_{1}(x)\right)=$ $\partial \varphi_{1}(x)$ is the subdifferential of $\varphi_{1}$ at $x$, and $M_{2}\left(y, f_{2}(y)\right)=\partial \varphi_{2}(y)$ is the subdifferential of $\varphi_{2}$ at $y$, then the problem (4.7) reduces to the following system of variational inequalities, which is to find $\left(x_{1}, x_{2}\right) \in E_{1} \times E_{2}$ such that

$$
\begin{aligned}
& \left\langle F_{1}\left(x_{1}, x_{2}\right), a-x_{1}\right\rangle+\varphi_{1}(a)-\varphi_{1}\left(x_{1}\right) \geq 0, \quad \forall a \in E_{1}, \\
& \left\langle F_{2}\left(x_{1}, x_{2}\right), b-x_{2}\right\rangle+\varphi_{2}(b)-\varphi_{2}\left(x_{2}\right) \geq 0, \quad \forall b \in E_{2} .
\end{aligned}
$$

Problem (4.8) was introduced and studied by Cho et al. [23].

If $M_{1}\left(x, f_{1}(x)\right)=\partial \delta_{k_{1}}(x), M_{2}\left(y, f_{2}(y)\right)=\partial \delta_{k_{2}}(y)$, for all $x \in K_{1}, y \in K_{2}$, where $K_{1} \subset E_{1}, K_{2} \subset E_{2}$ are two nonempty, closed, and convex subsets and $\partial \delta_{k_{1}}$ and $\partial \delta_{k_{2}}$ denote the indicator functions of $K_{1}, K_{2}$, respectively, then problem (4.8) reduces to the following system of variational inequalities, which is to find $\left(x_{1}, x_{2}\right) \in E_{1} \times E_{2}$ such that

$$
\begin{aligned}
& \left\langle F_{1}\left(x_{1}, x_{2}\right), a-x_{1}\right\rangle \geq 0, \quad \forall a \in K_{1}, \\
& \left\langle F_{2}\left(x_{1}, x_{2}\right), b-x_{2}\right\rangle \geq 0, \quad \forall b \in K_{2} .
\end{aligned}
$$

Problem (4.9) is just the problem [24] with $F_{1}$ and $F_{2}$ being single-valued.

If $E_{1}=E_{2}=\mathscr{\ell}$ is a Hilbert space and $K_{1}=K_{2}=K$ is a nonempty, closed, and convex subset, $F_{1}(x, y)=\rho T(y, x)+x-y$ and $F_{2}(x, y)=\lambda T(x, y)+y-x$, for all $x, y \in K$, where $T: K \times K \rightarrow \mathscr{H}$ is a mapping on $K \times K, \rho, \lambda>0$ are two numbers, then problem (4.9) reduces to the following problem, which is to find $x_{1}, x_{2} \in K$ such that

$$
\begin{aligned}
& \left\langle\rho T\left(x_{2}, x_{1}\right)+x_{1}-x_{2}, a-x_{1}\right\rangle \geq 0, \quad \forall a \in K, \\
& \left\langle\lambda T\left(x_{1}, x_{2}\right)+x_{2}-x_{1}, a-x_{2}\right\rangle \geq 0, \quad \forall a \in K .
\end{aligned}
$$

Problem (4.10) was introduced and studied by Verma [25]. 
Lemma 4.1. Let, for $i=1,2,3, \phi_{i}: E_{i} \rightarrow E_{i}$ be a single-valued mapping satisfying $\phi_{i}(x+y)=$ $\phi_{i}(x)+\phi_{i}(y)$ and $\operatorname{ker} \phi_{i}=\{0\}, \eta_{i}: E_{i} \times E_{i} \rightarrow E_{i}$ a single-valued mapping, $H_{i}: E_{i} \rightarrow E_{i} a$ strictly $\eta_{i}$-accretive mapping and $M_{i}: E_{i} \times E_{i} \rightarrow 2^{E_{i}} a\left(H_{i}, \phi_{i}\right)$ - $\eta_{i}$-accretive mapping in the second argument. Then $\left(x_{1}, x_{2}, x_{3}, u_{11}, u_{12}, u_{13}, u_{21}, u_{22}, u_{23}, u_{31}, u_{32}, u_{33}\right)$ in which $x_{i} \in E_{i}, u_{1 i} \in T_{1 i}\left(x_{1}\right)$, $u_{2 i} \in T_{2 i}\left(x_{2}\right), u_{3 i} \in T_{3 i}\left(x_{3}\right)(i=1,2,3)$ is a solution of the problem (4.1) if and only if

$$
\begin{aligned}
f_{i}\left(x_{i}\right)=J_{M_{i}\left(x_{i},\right), \phi_{i}}^{H_{i}, \eta_{i}} & H_{i} \circ f_{i}\left(x_{i}\right)-\phi_{i} \circ F_{i}\left(x_{1}-p_{1}\left(x_{1}\right), x_{2}-p_{2}\left(x_{2}\right), x_{3}-p_{3}\left(x_{3}\right)\right) \\
& \left.-\phi_{i} \circ N_{i}\left(u_{i 1}, u_{i 2}, u_{i 3}\right)\right],
\end{aligned}
$$

where $J_{M_{i}\left(x_{i},\right), \phi_{i}}^{H_{i}, \eta_{i}}=\left(H_{i}+\phi_{i} \circ M_{i}\left(x_{i}, \cdot\right)\right)^{-1}$.

Proof. The fact directly follows from Definition 3.6.

Algorithm 4.2. For any $x_{i}^{0} \in E_{i}$, take $u_{1 i}^{0} \in T_{1 i}\left(x_{1}^{0}\right), u_{2 i}^{0} \in T_{2 i}\left(x_{2}^{0}\right)$, and $u_{3 i}^{0} \in T_{3 i}\left(x_{3}^{0}\right)(i=1,2,3)$. For $i=1,2,3$, let

$$
\begin{gathered}
x_{i}^{1}=x_{i}^{0}-f_{i}\left(x_{i}^{0}\right)+J_{M_{i}\left(x_{i}^{0},\right), \phi_{i}}^{H_{i}, \eta_{i}}\left[H_{i} \circ f_{i}\left(x_{i}^{0}\right)-\phi_{i} \circ F_{i}\left(x_{1}^{0}-p_{1}\left(x_{1}^{0}\right), x_{2}^{0}-p_{2}\left(x_{2}^{0}\right), x_{3}^{0}-p_{3}\left(x_{3}^{0}\right)\right)\right. \\
\left.-\phi_{i} \circ N_{i}\left(u_{i 1}^{0}, u_{i 2}^{0}, u_{i 3}^{0}\right)\right] .
\end{gathered}
$$

Since $u_{1 i}^{0} \in T_{1 i}\left(x_{1}^{0}\right), u_{2 i}^{0} \in T_{2 i}\left(x_{2}^{0}\right), u_{3 i}^{0} \in T_{3 i}\left(x_{3}^{0}\right)(i=1,2,3)$, by Nodler's theorem [26], there exist $u_{1 i}^{1} \in T_{1 i}\left(x_{1}^{1}\right), u_{2 i}^{1} \in T_{2 i}\left(x_{2}^{1}\right), u_{3 i}^{1} \in T_{3 i}\left(x_{3}^{1}\right)(i=1,2,3)$, such that, for each $i=1,2,3$,

$$
\begin{aligned}
& \left\|u_{1 i}^{1}-u_{1 i}^{0}\right\| \leq(1+1) \widetilde{H}\left(T_{1 i}\left(x_{1}^{1}\right), T_{1 i}\left(x_{1}^{0}\right)\right), \\
& \left\|u_{2 i}^{1}-u_{2 i}^{0}\right\| \leq(1+1) \widetilde{H}\left(T_{2 i}\left(x_{2}^{1}\right), T_{2 i}\left(x_{2}^{0}\right)\right), \\
& \left\|u_{3 i}^{1}-u_{3 i}^{0}\right\| \leq(1+1) \widetilde{H}\left(T_{3 i}\left(x_{3}^{1}\right), T_{3 i}\left(x_{3}^{0}\right)\right) .
\end{aligned}
$$

For $i=1,2,3$, let

$$
\begin{aligned}
x_{i}^{2}=x_{i}^{1}-f_{i}\left(x_{i}^{1}\right)+J_{M_{i}\left(x_{i}^{1},\right), \phi_{i}}^{H_{i}, \eta_{i}} & {\left[H_{i} \circ f_{i}\left(x_{i}^{1}\right)-\phi_{i} \circ F_{i}\left(x_{1}^{1}-p_{1}\left(x_{1}^{1}\right), x_{2}^{1}-p_{2}\left(x_{2}^{1}\right), x_{3}^{1}-p_{3}\left(x_{3}^{1}\right)\right)\right.} \\
& \left.-\phi_{i} \circ N_{i}\left(u_{i 1}^{1}, u_{i 2}^{1}, u_{i 3}^{1}\right)\right] .
\end{aligned}
$$

Again by Nodler's theorem [26], there exist $u_{1 i}^{2} \in T_{1 i}\left(x_{1}^{2}\right), u_{2 i}^{2} \in T_{2 i}\left(x_{2}^{2}\right), u_{3 i}^{2} \in T_{3 i}\left(x_{3}^{2}\right)(i=$ $1,2,3)$, such that, for each $i=1,2,3$,

$$
\begin{aligned}
& \left\|u_{1 i}^{2}-u_{1 i}^{1}\right\| \leq\left(1+\frac{1}{2}\right) \widetilde{H}\left(T_{1 i}\left(x_{1}^{2}\right), T_{1 i}\left(x_{1}^{1}\right)\right), \\
& \left\|u_{2 i}^{2}-u_{2 i}^{1}\right\| \leq\left(1+\frac{1}{2}\right) \widetilde{H}\left(T_{2 i}\left(x_{2}^{2}\right), T_{2 i}\left(x_{2}^{1}\right)\right), \\
& \left\|u_{3 i}^{2}-u_{3 i}^{1}\right\| \leq\left(1+\frac{1}{2}\right) \widetilde{H}\left(T_{3 i}\left(x_{3}^{2}\right), T_{3 i}\left(x_{3}^{1}\right)\right) .
\end{aligned}
$$


By induction, we can compute the sequences $x_{i}^{n}, u_{1 i}^{n}, u_{2 i}^{n}, u_{3 i}^{n}(i=1,2,3)$ by the following iterative schemes such that, for each $i=1,2,3$,

$$
\begin{aligned}
& x_{i}^{n+1}=x_{i}^{n}-f_{i}\left(x_{i}^{n}\right)+J_{M_{i}\left(x_{i}^{n},\right), \phi_{i}}^{H_{i}, \eta_{i}}\left[H_{i} \circ f_{i}\left(x_{i}^{n}\right)-\phi_{i} \circ F_{i}\left(x_{1}^{n}-p_{1}\left(x_{1}^{n}\right), x_{2}^{n}-p_{2}\left(x_{2}^{n}\right), x_{3}^{n}-p_{3}\left(x_{3}^{n}\right)\right)\right. \\
&\left.-\phi_{i} \circ N_{i}\left(u_{i 1}^{n}, u_{i 2}^{n}, u_{i 3}^{n}\right)\right], \\
& u_{1 i}^{n} \in T_{1 i}\left(x_{1}^{n}\right), \quad\left\|u_{1 i}^{n}-u_{1 i}^{n-1}\right\| \leq\left(1+\frac{1}{n}\right) \widetilde{H}\left(T_{1 i}\left(x_{1}^{n}\right), T_{1 i}\left(x_{1}^{n-1}\right)\right), \\
& u_{2 i}^{n} \in T_{2 i}\left(x_{2}^{n}\right), \quad\left\|u_{2 i}^{n}-u_{2 i}^{n-1}\right\| \leq\left(1+\frac{1}{n}\right) \widetilde{H}\left(T_{2 i}\left(x_{2}^{n}\right), T_{2 i}\left(x_{2}^{n-1}\right)\right), \\
& u_{3 i}^{n} \in T_{3 i}\left(x_{3}^{n}\right), \quad\left\|u_{3 i}^{n}-u_{3 i}^{n-1}\right\| \leq\left(1+\frac{1}{n}\right) \widetilde{H}\left(T_{3 i}\left(x_{3}^{n}\right), T_{3 i}\left(x_{3}^{n-1}\right)\right),
\end{aligned}
$$

for all $n=0,1,2, \ldots$

Now we prove the existence of solution of the SGVIP (4.1) and the convergence of Algorithm 4.2.

Theorem 4.3. Let, for $i=1,2,3, E_{i}$ be a q-uniformly smooth Banach space and let $\phi_{i}: E_{i} \rightarrow E_{i}$ be a $\theta_{i^{-}}$ Lipschitz continuous mapping satisfying $\phi_{i}(x+y)=\phi_{i}(x)+\phi_{i}(y)$ and $\operatorname{ker} \phi_{i}=\{0\}$. Let $\eta_{i}: E_{i} \times E_{i} \rightarrow$ $E_{i}$ be $\tau_{i}$-Lipschitz continuous, let $H_{i}: E_{i} \rightarrow E_{i}$ be a $\gamma_{i}$-strongly $\eta_{i}$-accretive and $s_{i}$-Lipschitz continuous mapping, $f_{i}: E_{i} \rightarrow E_{i}$ be $\left(\alpha_{i}, \mu_{i}\right)$-relaxed cocoercive and $\xi_{i}$-Lipschitz continuous, and let $p_{i}: E_{i} \rightarrow E_{i}$ be a strongly accretive mapping with constant $\delta_{p_{i}}$ and Lipschitz continuous with constant $\lambda_{p_{i}}$. Suppose that $F_{i}: \prod_{k=1}^{3} E_{k} \rightarrow E_{i}$ is $\beta_{i k}$-Lipschitz continuous in the kth argument and $N_{i}$ : $\prod_{k=1}^{3} E_{k} \rightarrow E_{i}$ be $\zeta_{i k}$-Lipschitz continuous in the kth argument for $k=1,2,3, M_{i}: E_{i} \times E_{i} \rightarrow 2^{E_{i}}$ is a $\left(H_{i}, \phi_{i}\right)$ - $\eta_{i}$-accretive mapping in the second argument, and set-valued mappings $T_{1 i}: E_{1} \rightarrow C B\left(E_{i}\right)$, $T_{2 i}: E_{2} \rightarrow C B\left(E_{i}\right), T_{3 i}: E_{3} \rightarrow C B\left(E_{i}\right)$ are $\widetilde{H}$-Lipschitz continuous with constants $t_{1 i}>0, t_{2 i}>0$, $t_{3 i}>0$, respectively. In addition if for all $i=1,2,3$, one has

$$
\begin{gathered}
\left\|J_{M_{i}\left(x_{i 1}, \cdot\right), \phi_{i}}^{H_{i}, \eta_{i}}\left(z_{i}\right)-J_{M_{i}\left(x_{i 2}, \cdot\right), \phi_{i}}^{H_{i}, \eta_{i}}\left(z_{i}\right)\right\| \leq \delta_{i}\left\|x_{i 1}-x_{i 2}\right\|, \quad \forall x_{i 1}, x_{i 2}, z_{i} \in E_{i}, \\
0<\left(1+q \alpha_{1} \xi_{1}^{q}-q \mu_{1}+c_{q} \xi_{1}^{q}\right)^{1 / q}+\delta_{1}+\frac{\tau_{1}^{q-1}}{\gamma_{1}} \\
\times\left(\left(s_{1}^{q} \xi_{1}^{q}+q \theta_{1} \beta_{11} s_{1}^{q-1} \xi_{1}^{q-1}\left(1-q \delta_{p_{1}}+c_{q} \lambda_{p_{1}}^{q}\right)^{1 / q}+c_{q} \theta_{1}^{q} \beta_{11}^{q}\left(1-q \delta_{p_{1}}+c_{q} \lambda_{P_{1}}^{q}\right)\right)^{1 / q}\right. \\
\left.+\sum_{j=1}^{3} \theta_{1} \zeta_{1 j} t_{1 j}\right)+\left(\theta_{2} \beta_{21} \frac{\tau_{2}^{q-1}}{\gamma_{2}}+\theta_{3} \beta_{31} \frac{\tau_{3}^{q-1}}{\gamma_{3}}\right)\left(1-q \delta_{p_{1}}+c_{q} \lambda_{P_{1}}^{q}\right)^{1 / q}<1, \\
0<\left(1+q \alpha_{2} \xi_{2}^{q}-q \mu_{2}+c_{q} \xi_{2}^{q}\right)^{1 / q}+\delta_{2}+\frac{\tau_{2}^{q-1}}{\gamma_{2}}
\end{gathered}
$$




$$
\begin{aligned}
\times & \left(\left(s_{2}^{q} \xi_{2}^{q}+q \theta_{2} \beta_{22} s_{2}^{q-1} \xi_{2}^{q-1}\left(1-q \delta_{p_{2}}+c_{q} \lambda_{p_{2}}^{q}\right)^{1 / q}+c_{q} \theta_{2}^{q} \beta_{22}^{q}\left(1-q \delta_{p_{2}}+c_{q} \lambda_{P_{2}}^{q}\right)\right)^{1 / q}\right. \\
& \left.+\sum_{j=1}^{3} \theta_{2} \zeta_{2 j} t_{2 j}\right)+\left(\theta_{1} \beta_{12} \frac{\tau_{1}^{q-1}}{\gamma_{1}}+\theta_{3} \beta_{32} \frac{\tau_{3}^{q-1}}{\gamma_{3}}\right)\left(1-q \delta_{p_{2}}+c_{q} \lambda_{P_{2}}^{q}\right)^{1 / q}<1, \\
0< & \left(1+q \alpha_{3} \xi_{3}^{q}-q \mu_{3}+c_{q} \xi_{3}^{q}\right)^{1 / q}+\delta_{3}+\frac{\tau_{3}^{q-1}}{\gamma_{3}} \\
\times & \left(\left(s_{3}^{q} \xi_{3}^{q}+q \theta_{3} \beta_{33} s_{3}^{q-1} \xi_{3}^{q-1}\left(1-q \delta_{p_{3}}+c_{q} \lambda_{p_{3}}^{q}\right)^{1 / q}+c_{q} \theta_{3}^{q} \beta_{33}^{q}\left(1-q \delta_{p_{3}}+c_{q} \lambda_{P_{3}}^{q}\right)\right)^{1 / q}\right. \\
& \left.+\sum_{j=1}^{3} \theta_{3} \zeta_{3 j} t_{3 j}\right)+\left(\theta_{1} \beta_{13} \frac{\tau_{1}^{q-1}}{\gamma_{1}}+\theta_{2} \beta_{23} \frac{\tau_{2}^{q-1}}{\gamma_{2}}\right)\left(1-q \delta_{p_{3}}+c_{q} \lambda_{P_{3}}^{q}\right)^{1 / q}<1 .
\end{aligned}
$$

Then the problem (4.1) admits a solution $\left(x_{1}, x_{2}, x_{3}, u_{11}, u_{12}, u_{13}, u_{21}, u_{22}, u_{23}, u_{31}, u_{32}, u_{33}\right)$ and sequences $x_{1}^{n}, x_{2}^{n}, x_{3}^{n}, u_{11}^{n}, u_{12}^{n}, u_{13}^{n}, u_{21}^{n}, u_{22}^{n}, u_{23}^{n}, u_{31}^{n}, u_{32}^{n}, u_{33}^{n}$ converge to $x_{1}, x_{2}, x_{3}, u_{11}, u_{12}, u_{13}, u_{21}$, $u_{22}, u_{23}, u_{31}, u_{32}, u_{33}$, respectively, where $x_{i}^{n}, u_{1 i}^{n}, u_{2 i}^{n}, u_{3 i}^{n}(i=1,2,3)$ are sequences generated by Algorithm 4.2.

Proof. For $i=1,2,3$, let

$$
D_{i}^{n}=H_{i} \circ f_{i}\left(x_{i}^{n}\right)-\phi_{i} \circ F_{i}\left(x_{1}^{n}-p_{1}\left(x_{1}^{n}\right), x_{2}^{n}-p_{2}\left(x_{2}^{n}\right), x_{3}^{n}-p_{3}\left(x_{3}^{n}\right)\right)-\phi_{i} \circ N_{i}\left(u_{i 1}^{n}, u_{i 2}^{n}, u_{i 3}^{n}\right) .
$$

By Algorithm 4.2 and (4.17), we have

$$
\begin{aligned}
& \left\|x_{i}^{n+1}-x_{i}^{n}\right\| \\
& =\| x_{i}^{n}-f_{i}\left(x_{i}^{n}\right)+J_{M_{i}\left(x_{i}^{n},\right), \phi_{i}}^{H_{i}, \eta_{i}}\left[H_{i} \circ f_{i}\left(x_{i}^{n}\right)-\phi_{i} \circ F_{i}\left(x_{1}^{n}-p_{1}\left(x_{1}^{n}\right), x_{2}^{n}-p_{2}\left(x_{2}^{n}\right), x_{3}^{n}-p_{3}\left(x_{3}^{n}\right)\right)\right. \\
& \left.-\phi_{i} \circ N_{i}\left(u_{i 1}^{n}, u_{i 2}^{n}, u_{i 3}^{n}\right)\right] \\
& -\left(x_{i}^{n-1}-f_{i}\left(x_{i}^{n-1}\right)\right. \\
& +J_{M_{i}\left(x_{i}^{n-1},\right), \phi_{i}}^{H_{i} \eta_{i}}\left[H_{i} \circ f_{i}\left(x_{i}^{n-1}\right)\right. \\
& -\phi_{i} \circ F_{i}\left(x_{1}^{n-1}-p_{1}\left(x_{1}^{n-1}\right), x_{2}^{n-1}-p_{2}\left(x_{2}^{n-1}\right), x_{3}^{n-1}-p_{3}\left(x_{3}^{n-1}\right)\right) \\
& \left.\left.-\phi_{i} \circ N_{i}\left(u_{i 1}^{n-1}, u_{i 2}^{n-1}, u_{i 3}^{n-1}\right)\right]\right) \| \\
& \leq\left\|x_{i}^{n}-x_{i}^{n-1}-\left[f_{i}\left(x_{i}^{n}\right)-f_{i}\left(x_{i}^{n-1}\right)\right]\right\|+\left\|J_{M_{i}\left(x_{i}^{n},\right)_{,}, \phi_{i}}^{H_{i}, \eta_{i}}\left(D_{i}^{n}\right)-J_{M_{i}\left(x_{i}^{n},\right)_{,}, \phi_{i}}^{H_{i}, \eta_{i}}\left(D_{i}^{n-1}\right)\right\|
\end{aligned}
$$




$$
\begin{aligned}
& +\left\|J_{M_{i}\left(x_{i}^{n},\right), \phi_{i}}^{H_{i}, \eta_{i}}\left(D_{i}^{n-1}\right)-J_{M_{i}\left(x_{i}^{n-1},\right), \phi_{i}}^{H_{i}, \eta_{i}}\left(D_{i}^{n-1}\right)\right\| \\
\leq & \left\|x_{i}^{n}-x_{i}^{n-1}-\left[f_{i}\left(x_{i}^{n}\right)-f_{i}\left(x_{i}^{n-1}\right)\right]\right\|+\frac{\tau_{i}^{q-1}}{\gamma_{i}}\left\|D_{i}^{n}-D_{i}^{n-1}\right\|+\delta_{i}\left\|x_{i}^{n}-x_{i}^{n-1}\right\| .
\end{aligned}
$$

Since $f_{i}: E_{i} \rightarrow E_{i}$ is $\left(\alpha_{i}, \mu_{i}\right)$-relaxed cocoercive and $\xi_{i}$-Lipschitz continuous and $p_{i}: E_{i} \rightarrow E_{i}$ is $\delta_{p_{i}}$-strongly accretive and $\lambda_{p_{i}}$-Lipschitz continuous, we have

$$
\begin{aligned}
& \left\|x_{i}^{n}-x_{i}^{n-1}-\left[f_{i}\left(x_{i}^{n}\right)-f_{i}\left(x_{i}^{n-1}\right)\right]\right\|^{q} \\
& \quad \leq\left\|x_{i}^{n}-x_{i}^{n-1}\right\|^{q}-q\left\langle f_{i}\left(x_{i}^{n}\right)-f_{i}\left(x_{i}^{n-1}\right), J_{q}\left(x_{i}^{n}-x_{i}^{n-1}\right)\right\rangle+c_{q}\left\|f_{i}\left(x_{i}^{n}\right)-f_{i}\left(x_{i}^{n-1}\right)\right\|^{q} \\
& \quad \leq\left(1+q \alpha_{i} \xi_{i}^{q}-q \mu_{i}+c_{q} \xi_{i}^{q}\right)\left\|x_{i}^{n}-x_{i}^{n-1}\right\|^{q}, \\
& \left\|x_{i}^{n}-x_{i}^{n-1}-\left[p_{i}\left(x_{i}^{n}\right)-p_{i}\left(x_{i}^{n-1}\right)\right]\right\|^{q} \\
& \quad \leq\left\|x_{i}^{n}-x_{i}^{n-1}\right\|^{q}-q\left\langle p_{i}\left(x_{i}^{n}\right)-p_{i}\left(x_{i}^{n-1}\right), J_{q}\left(x_{i}^{n}-x_{i}^{n-1}\right)\right\rangle+c_{q}\left\|p_{i}\left(x_{i}^{n}\right)-p_{i}\left(x_{i}^{n-1}\right)\right\|^{q} \\
& \quad \leq\left(1-q \delta_{p_{i}}+c_{q} \lambda_{p_{i}}^{q}\right)\left\|x_{i}^{n}-x_{i}^{n-1}\right\|^{q} .
\end{aligned}
$$

From (4.19), we have

$$
\begin{aligned}
& \left\|D_{i}^{n}-D_{i}^{n-1}\right\| \\
& =\| H_{i} \circ f_{i}\left(x_{i}^{n}\right)-\phi_{i} \circ F_{i}\left(x_{1}^{n}-p_{1}\left(x_{1}^{n}\right), x_{2}^{n}-p_{2}\left(x_{2}^{n}\right), x_{3}^{n}-p_{3}\left(x_{3}^{n}\right)\right)-\phi_{i} \circ N_{i}\left(u_{i 1}^{n}, u_{i 2}^{n}, u_{i 3}^{n}\right) \\
& -\left(H_{i} \circ f_{i}\left(x_{i}^{n-1}\right)-\phi_{i} \circ F_{i}\left(x_{1}^{n-1}-p_{1}\left(x_{1}^{n-1}\right), x_{2}^{n-1}-p_{2}\left(x_{2}^{n-1}\right), x_{3}^{n-1}-p_{3}\left(x_{3}^{n-1}\right)\right)\right. \\
& \left.-\phi_{i} \circ N_{i}\left(u_{i 1}^{n-1}, u_{i 2}^{n-1}, u_{i 3}^{n-1}\right)\right) \| \\
& \leq \| H_{i} \circ f_{i}\left(x_{i}^{n}\right)-H_{i} \circ f_{i}\left(x_{i}^{n-1}\right)-\left(\phi_{i} \circ F_{i}\left(x_{1}^{n}-p_{1}\left(x_{1}^{n}\right), x_{2}^{n}-p_{2}\left(x_{2}^{n}\right), x_{3}^{n}-p_{3}\left(x_{3}^{n}\right)\right)\right. \\
& \left.-\phi_{i} \circ F_{i}\left(x_{1}^{n-1}-p_{1}\left(x_{1}^{n-1}\right), x_{2}^{n-1}-p_{2}\left(x_{2}^{n-1}\right), x_{3}^{n-1}-p_{3}\left(x_{3}^{n-1}\right)\right)\right) \| \\
& +\left\|\phi_{i} \circ N_{i}\left(u_{i 1}^{n}, u_{i 2}^{n}, u_{i 3}^{n}\right)-\phi_{i} \circ N_{i}\left(u_{i 1}^{n-1}, u_{i 2}^{n-1}, u_{i 3}^{n-1}\right)\right\| \text {. }
\end{aligned}
$$


Since $F_{i}: \prod_{k=1}^{3} E_{k} \rightarrow E_{i}$ is $\beta_{i k}$-Lipschitz continuous in the $k$ th argument and by continuity of $\mathrm{H}_{i}, f_{i}, \phi_{i}(i=1,2,3)$, we have

$$
\begin{aligned}
\| H_{i} \circ f_{i}\left(x_{i}^{n}\right)-H_{i} \circ f_{i}\left(x_{i}^{n-1}\right)-\left(\phi_{i} \circ F_{i}\left(x_{1}^{n}-p_{1}\left(x_{1}^{n}\right), x_{2}^{n}-p_{2}\left(x_{2}^{n}\right), x_{3}^{n}-p_{3}\left(x_{3}^{n}\right)\right)\right. \\
\left.-\phi_{i} \circ F_{i}\left(x_{1}^{n-1}-p_{1}\left(x_{1}^{n-1}\right), x_{2}^{n-1}-p_{2}\left(x_{2}^{n-1}\right), x_{3}^{n-1}-p_{3}\left(x_{3}^{n-1}\right)\right)\right) \| \\
\leq\left(s_{i}^{q} \xi_{i}^{q}+q \theta_{i} \beta_{i i} s_{i}^{q-1} \xi_{i}^{q-1}\left(1-q \delta_{p_{i}}+c_{q} \lambda_{p_{i}}^{q}\right)^{1 / q}+c_{q} \theta_{i}^{q} \beta_{i i}^{q}\left(1-q \delta_{p_{i}}+c_{q} \lambda_{P_{i}}^{q}\right)\right)^{1 / q}\left\|x_{i}^{n}-x_{i}^{n-1}\right\| \\
\quad+\sum_{j=1}^{i-1} \theta_{i} \beta_{i j}\left(1-q \delta_{p_{j}}+c_{q} \lambda_{P_{j}}^{q}\right)^{1 / q}\left\|x_{j}^{n}-x_{j}^{n-1}\right\|+\sum_{j=i+1}^{3} \theta_{i} \beta_{i j}\left(1-q \delta_{p_{j}}+c_{q} \lambda_{P_{j}}^{q}\right)^{1 / q}\left\|x_{j}^{n}-x_{j}^{n-1}\right\| .
\end{aligned}
$$

It follows from the Lipschitz continuity of $N_{i}$ and the $\widetilde{H}$-Lipschitz continuity of $T_{i j}$ that

$$
\begin{aligned}
&\left\|N_{i}\left(u_{i 1}^{n}, u_{i 2}^{n}, u_{i 3}^{n}\right)-N_{i}\left(u_{i 1}^{n-1}, u_{i 2}^{n-1}, u_{i 3}^{n-1}\right)\right\| \\
& \leq\left\|N_{i}\left(u_{i 1}^{n}, u_{i 2}^{n}, u_{i 3}^{n}\right)-N_{i}\left(u_{i 1}^{n-1}, u_{i 2}^{n}, u_{i 3}^{n}\right)\right\| \\
&+\left\|N_{i}\left(u_{i 1}^{n-1}, u_{i 2}^{n}, u_{i 3}^{n}\right)-N_{i}\left(u_{i 1}^{n-1}, u_{i 2}^{n-1}, u_{i 3}^{n}\right)\right\| \\
&+\left\|N_{i}\left(u_{i 1}^{n-1}, u_{i 2}^{n-1}, u_{i 3}^{n}\right)-N_{i}\left(u_{i 1}^{n-1}, u_{i 2}^{n-1}, u_{i 3}^{n-1}\right)\right\| \\
& \leq \zeta_{i 1}\left\|u_{i 1}^{n}-u_{i 1}^{n-1}\right\|+\zeta_{i 2}\left\|u_{i 2}^{n}-u_{i 2}^{n-1}\right\|+\zeta_{i 3}\left\|u_{i 3}^{n}-u_{i 3}^{n-1}\right\| \\
&= \sum_{j=1}^{3} \zeta_{i j}\left\|u_{i j}^{n}-u_{i j}^{n-1}\right\| \leq \sum_{j=1}^{3} \zeta_{i j}\left(1+\frac{1}{n}\right) \tilde{\mathrm{H}}\left(T_{i j}\left(x_{i}^{n}\right), T_{i j}\left(x_{i}^{n-1}\right)\right) \\
& \leq \sum_{j=1}^{3} \zeta_{i j} t_{i j}\left(1+\frac{1}{n}\right)\left\|x_{i}^{n}-x_{i}^{n-1}\right\|, \quad i=1,2,3 .
\end{aligned}
$$

It follows from (4.20)-(4.24) that, for each $i=1,2,3$,

$$
\begin{aligned}
& \left\|x_{i}^{n+1}-x_{i}^{n}\right\| \\
& \leq\left[\left(1+q \alpha_{i} \xi_{i}^{q}-q \mu_{i}+c_{q} \xi_{i}^{q}\right)^{1 / q}+\delta_{i}\right. \\
& \quad+\frac{\tau_{i}^{q-1}}{\gamma_{i}}\left(\left(s_{i}^{q} \xi_{i}^{q}+q \theta_{i} \beta_{i i} s_{i}^{q-1} \xi_{i}^{q-1}\left(1-q \delta_{p_{i}}+c_{q} \lambda_{p_{i}}^{q}\right)^{1 / q}\right.\right.
\end{aligned}
$$


ISRN Applied Mathematics

$$
\begin{array}{r}
\left.\left.\left.+c_{q} \theta_{i}^{q} \beta_{i i}^{q}\left(1-q \delta_{p_{i}}+c_{q} \lambda_{P_{i}}^{q}\right)\right)^{1 / q}+\sum_{j=1}^{3} \theta_{i} \zeta_{i j} t_{i j}\left(1+\frac{1}{n}\right)\right)\right] \\
\times\left\|x_{i}^{n}-x_{i}^{n-1}\right\|+\sum_{j=1}^{i-1} \theta_{i} \beta_{i j} \frac{\tau_{i}^{q-1}}{\gamma_{i}}\left(1-q \delta_{p_{j}}+c_{q} \lambda_{P_{j}}^{q}\right)^{1 / q}\left\|x_{j}^{n}-x_{j}^{n-1}\right\| \\
+\sum_{j=i+1}^{3} \theta_{i} \beta_{i j} \frac{\tau_{i}^{q-1}}{\gamma_{i}}\left(1-q \delta_{p_{j}}+c_{q} \lambda_{P_{j}}^{q}\right)^{1 / q}\left\|x_{j}^{n}-x_{j}^{n-1}\right\| .
\end{array}
$$

Therefore,

$$
\begin{aligned}
& \sum_{i=1}^{3}\left\|x_{i}^{n+1}-x_{i}^{n}\right\| \\
& \leq \sum_{i=1}^{3}\left\{\left[\left(1+q \alpha_{i} \xi_{i}^{q}-q \mu_{i}+c_{q} \xi_{i}^{q}\right)^{1 / q}+\delta_{i}\right.\right. \\
& +\frac{\tau_{i}^{q-1}}{r_{i}}\left(\left(s_{i}^{q} \xi_{i}^{q}+q \theta_{i} \beta_{i i} s_{i}^{q-1} \xi_{i}^{q-1}\left(1-q \delta_{p_{i}}+c_{q} \lambda_{p_{i}}^{q}\right)^{1 / q}+c_{q} \theta_{i}^{q} \beta_{i i}^{q}\right.\right. \\
& \left.\left.\left.\times\left(1-q \delta_{p_{i}}+c_{q} \lambda_{P_{i}}^{q}\right)\right)^{1 / q}+\sum_{j=1}^{3} \theta_{i} \zeta_{i j} t_{i j}\left(1+\frac{1}{n}\right)\right)\right] \\
& \times\left\|x_{i}^{n}-x_{i}^{n-1}\right\|+\sum_{j=1}^{i-1} \theta_{i} \beta_{i j} \frac{\tau_{i}^{q-1}}{\gamma_{i}}\left(1-q \delta_{p_{j}}+c_{q} \lambda_{P_{j}}^{q}\right)^{1 / q}\left\|x_{j}^{n}-x_{j}^{n-1}\right\| \\
& \left.+\sum_{j=i+1}^{3} \theta_{i} \beta_{i j} \frac{\tau_{i}^{q-1}}{\gamma_{i}}\left(1-q \delta_{p_{j}}+c_{q} \lambda_{P_{j}}^{q}\right)^{1 / q}\left\|x_{j}^{n}-x_{j}^{n-1}\right\|\right\} \\
& =\left[\left(1+q \alpha_{1} \xi_{1}^{q}-q \mu_{1}+c_{q} \xi_{1}^{q}\right)^{1 / q}+\delta_{1}+\frac{\tau_{1}^{q-1}}{\gamma_{1}}\right. \\
& \times\left(\left(s_{1}^{q} \xi_{1}^{q}+q \theta_{1} \beta_{11} s_{1}^{q-1} \xi_{1}^{q-1}\left(1-q \delta_{p_{1}}+c_{q} \lambda_{p_{1}}^{q}\right)^{1 / q}+c_{q} \theta_{1}^{q} \beta_{11}^{q}\left(1-q \delta_{p_{1}}+c_{q} \lambda_{P_{1}}^{q}\right)\right)^{1 / q}\right. \\
& \left.\left.+\sum_{j=1}^{3} \theta_{1} \zeta_{1 j} t_{1 j}\left(1+\frac{1}{n}\right)\right)+\left(\theta_{2} \beta_{21} \frac{\tau_{2}^{q-1}}{\gamma_{2}}+\theta_{3} \beta_{31} \frac{\tau_{3}^{q-1}}{\gamma_{3}}\right)\left(1-q \delta_{p_{1}}+c_{q} \lambda_{P_{1}}^{q}\right)^{1 / q}\right] \\
& \times\left\|x_{1}^{n}-x_{1}^{n-1}\right\|+\left[\left(1+q \alpha_{2} \xi_{2}^{q}-q \mu_{2}+c_{q} \xi_{2}^{q}\right)^{1 / q}+\delta_{2}+\frac{\tau_{2}^{q-1}}{\gamma_{2}}\right.
\end{aligned}
$$




$$
\begin{aligned}
& \times\left(\left(s_{2}^{q} \xi_{2}^{q}+q \theta_{2} \beta_{22} s_{2}^{q-1} \xi_{2}^{q-1}\left(1-q \delta_{p_{2}}+c_{q} \lambda_{p_{2}}^{q}\right)^{1 / q}\right.\right. \\
& \left.\left.+c_{q} \theta_{2}^{q} \beta_{22}^{q}\left(1-q \delta_{p_{2}}+c_{q} \lambda_{P_{2}}^{q}\right)\right)^{1 / q}+\sum_{j=1}^{3} \theta_{2} \zeta_{2 j} t_{2 j}\left(1+\frac{1}{n}\right)\right) \\
& \left.+\left(\theta_{1} \beta_{12} \frac{\tau_{1}^{q-1}}{\gamma_{1}}+\theta_{3} \beta_{32} \frac{\tau_{3}^{q-1}}{\gamma_{3}}\right)\left(1-q \delta_{p_{2}}+c_{q} \lambda_{P_{2}}^{q}\right)^{1 / q}\right] \\
& \times\left\|x_{2}^{n}-x_{2}^{n-1}\right\|+\left[\left(1+q \alpha_{3} \xi_{3}^{q}-q \mu_{3}+c_{q} \xi_{3}^{q}\right)^{1 / q}+\delta_{3}+\frac{\tau_{3}^{q-1}}{\gamma_{3}}\right. \\
& \times\left(\left(s_{3}^{q} \xi_{3}^{q}+q \theta_{3} \beta_{33} s_{3}^{q-1} \xi_{3}^{q-1}\left(1-q \delta_{p_{3}}+c_{q} \lambda_{p_{3}}^{q}\right)^{1 / q}\right.\right. \\
& \left.\left.+c_{q} \theta_{3}^{q} \beta_{33}^{q}\left(1-q \delta_{p_{3}}+c_{q} \lambda_{P_{3}}^{q}\right)\right)^{1 / q}+\sum_{j=1}^{3} \theta_{3} \zeta_{3 j} t_{3 j}\left(1+\frac{1}{n}\right)\right) \\
& \left.+\left(\theta_{1} \beta_{13} \frac{\tau_{1}^{q-1}}{\gamma_{1}}+\theta_{2} \beta_{23} \frac{\tau_{2}^{q-1}}{\gamma_{2}}\right)\left(1-q \delta_{p_{3}}+c_{q} \lambda_{P_{3}}^{q}\right)^{1 / q}\right] \\
& \times\left\|x_{3}^{n}-x_{3}^{n-1}\right\| \\
& \leq \theta_{n}\left(\sum_{i=1}^{n}\left\|x_{i}^{n}-x_{i}^{n-1}\right\|\right),
\end{aligned}
$$

where

$$
\begin{gathered}
\theta_{n}=\max \left\{\begin{array}{r}
\left(1+q \alpha_{1} \xi_{1}^{q}-q \mu_{1}+c_{q} \xi_{1}^{q}\right)^{1 / q}+\delta_{1}+\frac{\tau_{1}^{q-1}}{\gamma_{1}}\left(\left(s_{1}^{q} \xi_{1}^{q}+q \theta_{1} \beta_{11} s_{1}^{q-1} \xi_{1}^{q-1}\left(1-q \delta_{p_{1}}+c_{q} \lambda_{p_{1}}^{q}\right)^{1 / q}\right.\right. \\
\left.+c_{q} \theta_{1}^{q} \beta_{11}^{q}\left(1-q \delta_{p_{1}}+c_{q} \lambda_{P_{1}}^{q}\right)\right)^{1 / q} \\
\left.+\sum_{j=1}^{3} \theta_{1} \zeta_{1 j} t_{1 j}\left(1+\frac{1}{n}\right)\right)
\end{array}\right. \\
+\left(\theta_{2} \beta_{21} \frac{\tau_{2}^{q-1}}{\gamma_{2}}+\theta_{3} \beta_{31} \frac{\tau_{3}^{q-1}}{\gamma_{3}}\right)\left(1-q \delta_{p_{1}}+c_{q} \lambda_{P_{1}}^{q}\right)^{1 / q},
\end{gathered}
$$


ISRN Applied Mathematics

$$
\begin{aligned}
& \left(1+q \alpha_{2} \xi_{2}^{q}-q \mu_{2}+c_{q} \xi_{2}^{q}\right)^{1 / q}+\delta_{2}+\frac{\tau_{2}^{q-1}}{r_{2}}\left(\left(s_{2}^{q} \xi_{2}^{q}+q \theta_{2} \beta_{22} s_{2}^{q-1} \xi_{2}^{q-1}\left(1-q \delta_{p_{2}}+c_{q} \lambda_{p_{2}}^{q}\right)^{1 / q}\right.\right. \\
& \left.+c_{q} \theta_{2}^{q} \beta_{22}^{q}\left(1-q \delta_{p_{2}}+c_{q} \lambda_{P_{2}}^{q}\right)\right)^{1 / q} \\
& \left.+\sum_{j=1}^{3} \theta_{2} \zeta_{2 j} t_{2 j}\left(1+\frac{1}{n}\right)\right) \\
& +\left(\theta_{1} \beta_{12} \frac{\tau_{1}^{q-1}}{\gamma_{1}}+\theta_{3} \beta_{32} \frac{\tau_{3}^{q-1}}{\gamma_{3}}\right)\left(1-q \delta_{p_{2}}+c_{q} \lambda_{P_{2}}^{q}\right)^{1 / q}, \\
& \left(1+q \alpha_{3} \xi_{3}^{q}-q \mu_{3}+c_{q} \xi_{3}^{q}\right)^{1 / q}+\delta_{3}+\frac{\tau_{3}^{q-1}}{\gamma_{3}}\left(\left(s_{3}^{q} \xi_{3}^{q}+q \theta_{3} \beta_{33} s_{3}^{q-1} \xi_{3}^{q-1}\left(1-q \delta_{p_{3}}+c_{q} \lambda_{p_{3}}^{q}\right)^{1 / q}\right.\right. \\
& \left.+c_{q} \theta_{3}^{q} \beta_{33}^{q}\left(1-q \delta_{p_{3}}+c_{q} \lambda_{P_{3}}^{q}\right)\right)^{1 / q} \\
& \left.+\sum_{j=1}^{3} \theta_{3} \zeta_{3 j} t_{3 j}\left(1+\frac{1}{n}\right)\right) \\
& \left.+\left(\theta_{1} \beta_{13} \frac{\tau_{1}^{q-1}}{\gamma_{1}}+\theta_{2} \beta_{23} \frac{\tau_{2}^{q-1}}{\gamma_{2}}\right)\left(1-q \delta_{p_{3}}+c_{q} \lambda_{P_{3}}^{q}\right)^{1 / q}\right\} .
\end{aligned}
$$

Let

$$
\begin{aligned}
\theta=\max \{( & \left.+q \alpha_{1} \xi_{1}^{q}-q \mu_{1}+c_{q} \xi_{1}^{q}\right)^{1 / q}+\delta_{1}+\frac{\tau_{1}^{q-1}}{\gamma_{1}} \\
& \times\left(\left(s_{1}^{q} \xi_{1}^{q}+q \theta_{1} \beta_{11} s_{1}^{q-1} \xi_{1}^{q-1}\left(1-q \delta_{p_{1}}+c_{q} \lambda_{p_{1}}^{q}\right)^{1 / q}+c_{q} \theta_{1}^{q} \beta_{11}^{q}\left(1-q \delta_{p_{1}}+c_{q} \lambda_{P_{1}}^{q}\right)\right)^{1 / q}\right. \\
& \left.+\sum_{j=1}^{3} \theta_{1} \zeta_{1 j} t_{1 j}\right)+\left(\theta_{2} \beta_{21} \frac{\tau_{2}^{q-1}}{\gamma_{2}}+\theta_{3} \beta_{31} \frac{\tau_{3}^{q-1}}{\gamma_{3}}\right)\left(1-q \delta_{p_{1}}+c_{q} \lambda_{P_{1}}^{q}\right)^{1 / q}, \\
\left(1+q \alpha_{2} \xi_{2}^{q}-q \mu_{2}+c_{q} \xi_{2}^{q}\right)^{1 / q}+\delta_{2}+\frac{\tau_{2}^{q-1}}{\gamma_{2}} & \left(\left(s_{2}^{q} \xi_{2}^{q}+q \theta_{2} \beta_{22} s_{2}^{q-1} \xi_{2}^{q-1}\left(1-q \delta_{p_{2}}+c_{q} \lambda_{p_{2}}^{q}\right)^{1 / q}+c_{q} \theta_{2}^{q} \beta_{22}^{q}\left(1-q \delta_{p_{2}}+c_{q} \lambda_{P_{2}}^{q}\right)\right)^{1 / q}\right.
\end{aligned}
$$




$$
\begin{gathered}
\left.+\sum_{j=1}^{3} \theta_{2} \zeta_{2 j} t_{2 j}\right)+\left(\theta_{1} \beta_{12} \frac{\tau_{1}^{q-1}}{\gamma_{1}}+\theta_{3} \beta_{32} \frac{\tau_{3}^{q-1}}{\gamma_{3}}\right)\left(1-q \delta_{p_{2}}+c_{q} \lambda_{P_{2}}^{q}\right)^{1 / q} \\
\left(1+q \alpha_{3} \xi_{3}^{q}-q \mu_{3}+c_{q} \xi_{3}^{q}\right)^{1 / q}+\delta_{3}+\frac{\tau_{3}^{q-1}}{r_{3}} \\
\times\left(\left(s_{3}^{q} \xi_{3}^{q}+q \theta_{3} \beta_{33} s_{3}^{q-1} \xi_{3}^{q-1}\left(1-q \delta_{p_{3}}+c_{q} \lambda_{p_{3}}^{q}\right)^{1 / q}+c_{q} \theta_{3}^{q} \beta_{33}^{q}\left(1-q \delta_{p_{3}}+c_{q} \lambda_{P_{3}}^{q}\right)\right)^{1 / q}\right. \\
\left.\left.+\sum_{j=1}^{3} \theta_{3} \zeta_{3 j} t_{3 j}\right)+\left(\theta_{1} \beta_{13} \frac{\tau_{1}^{q-1}}{r_{1}}+\theta_{2} \beta_{23} \frac{\tau_{2}^{q-1}}{r_{2}}\right)\left(1-q \delta_{p_{3}}+c_{q} \lambda_{P_{3}}^{q}\right)^{1 / q}\right\} .
\end{gathered}
$$

We know that $\theta_{n} \rightarrow \theta$ as $n \rightarrow \infty$.

Define $\|\cdot\|_{*}$ on $E=\prod_{k=1}^{3} E_{k}$ by

$$
\left\|\left(z_{1}, z_{2}, z_{3}\right)\right\|_{*}=\left\|z_{1}\right\|+\left\|z_{2}\right\|+\left\|z_{3}\right\|, \quad \forall\left(z_{1}, z_{2}, z_{3}\right) \in E
$$

It is easy to see that $\left(E,\|\cdot\|_{*}\right)$ is a Banach space. Define $z^{n+1}=\left(x_{1}^{n+1}, x_{2}^{n+1}, x_{3}^{n+1}\right)$. Then we have

$$
\left\|z^{n+1}-z^{n}\right\|_{*}=\left\|x_{1}^{n+1}-x_{1}^{n}\right\|+\left\|x_{2}^{n+1}-x_{2}^{n}\right\|+\left\|x_{3}^{n+1}-x_{3}^{n}\right\|
$$

It follows from (4.18) that $0<\theta<1$, and hence there exists an $n_{0}>0$ and $\theta_{0} \in(0,1)$ such that $\theta_{n} \leq \theta_{0}$, for all $n \geq n_{0}$. Therefore, by (4.26) and (4.30), we have

$$
\left\|z^{n+1}-z^{n}\right\|_{*} \leq \theta_{0}^{n-n_{o}}\left\|z^{n_{0}+1}-z^{n_{0}}\right\|_{*}
$$

Hence, for any $m \geq n \geq n_{0}$, it follows that

$$
\begin{aligned}
\left\|x_{1}^{m}-x_{1}^{n}\right\| & \leq\left\|z^{m}-z^{n}\right\|_{*} \\
& \leq \sum_{i=n}^{m-1}\left\|z^{i+1}-z^{i}\right\|_{*} \\
& \leq \sum_{i=n}^{m-1} \theta_{0}^{i-n_{0}}\left\|z^{n_{0}+1}-z^{n_{0}}\right\|_{*} .
\end{aligned}
$$

Since $0<\theta_{0}<1$, it follows from (4.32) that $\left\|x_{1}^{m}-x_{1}^{n}\right\| \rightarrow 0$ as $n \rightarrow \infty$, and hence $\left\{x_{1}^{n}\right\}$ is a Cauchy sequence in $E_{1}$. By the same argument, we also have $\left\{x_{i}^{n}\right\}$ is a Cauchy sequence in $E_{i}(i=2,3)$. Thus, there exist $x_{1} \in E_{1}, x_{2} \in E_{2}, x_{3} \in E_{3}$ such that $x_{1}^{n} \rightarrow x_{1}, x_{2}^{n} \rightarrow x_{2}, x_{3}^{n} \rightarrow x_{3}$ as $n \rightarrow \infty$. 
Now we prove that $u_{1 i}^{n} \rightarrow u_{1 i} \in T_{1 i}\left(x_{1}\right), u_{2 i}^{n} \rightarrow u_{2 i} \in T_{2 i}\left(x_{2}\right), u_{3 i}^{n} \rightarrow u_{3 i} \in T_{3 i}\left(x_{3}\right)(i=$ $1,2,3)$. In fact, it follows from the Lipschitz continuity of $T_{1 i}, T_{2 i}, T_{3 i}$ that for, $i=1,2,3$,

$$
\begin{aligned}
& \left\|u_{1 i}^{n}-u_{1 i}^{n-1}\right\| \leq\left(1+\frac{1}{n}\right) t_{1 i}\left\|x_{1}^{n}-x_{1}^{n-1}\right\|, \\
& \left\|u_{2 i}^{n}-u_{2 i}^{n-1}\right\| \leq\left(1+\frac{1}{n}\right) t_{2 i}\left\|x_{2}^{n}-x_{2}^{n-1}\right\|, \\
& \left\|u_{3 i}^{n}-u_{3 i}^{n-1}\right\| \leq\left(1+\frac{1}{n}\right) t_{3 i}\left\|x_{3}^{n}-x_{3}^{n-1}\right\| .
\end{aligned}
$$

From (4.33), we have that $u_{1 i}^{n}, u_{2 i}^{n}, u_{3 i}^{n}(i=1,2,3)$ are also Cauchy sequences. Therefore, there exist $u_{1 i} \in E_{i}, u_{2 i} \in E_{i}, u_{3 i} \in E_{i}$ such that $u_{1 i}^{n} \rightarrow u_{1 i}, u_{2 i}^{n} \rightarrow u_{2 i}, u_{3 i}^{n} \rightarrow u_{3 i}$ as $n \rightarrow \infty$. Further, for $i=1,2,3$,

$$
\begin{aligned}
d\left(u_{1 i}, T_{1 i}\left(x_{1}\right)\right) & \leq\left\|u_{1 i}-u_{1 i}^{n}\right\|+d\left(u_{1 i}^{n}, T_{1 i}\left(x_{1}\right)\right) \\
& \leq\left\|u_{1 i}-u_{1 i}^{n}\right\|+\widetilde{H}\left(T_{1 i}\left(x_{1}^{n}\right), T_{1 i}\left(x_{1}\right)\right) \\
& \leq\left\|u_{1 i}-u_{1 i}^{n}\right\|+t_{1 i}\left\|x_{1}^{n}-x_{1}\right\| \longrightarrow 0 .
\end{aligned}
$$

Since $T_{1 i}\left(x_{1}\right)$ is closed, we have $u_{1 i} \in T_{1 i}\left(x_{1}\right)(i=1,2,3)$. Similarly $u_{2 i} \in T_{2 i}\left(x_{2}\right), u_{3 i} \in T_{3 i}\left(x_{3}\right)(i=$ $1,2,3)$. By continuity of $f_{i}, H_{i}, \phi_{i}, F_{i}, N_{i}, T_{1 i}, T_{2 i}, T_{3 i}, J_{M_{i}\left(x_{i}, \cdot\right), \phi_{i}}^{H_{i,}, l_{i}}$ and Algorithm 4.2, we know that $x_{1}, x_{2}, x_{3}, u_{11}, u_{12}, u_{13}, u_{21}, u_{22}, u_{23}, u_{31}, u_{32}, u_{33}$ satisfy the following relation:

$$
\begin{gathered}
f_{i}\left(x_{i}\right)=J_{M_{i}\left(x_{i},\right), \phi_{i}}^{H_{i,}, \eta_{i}}\left[H_{i} \circ f_{i}\left(x_{i}\right)-\phi_{i} \circ F_{i}\left(x_{1}-p_{1}\left(x_{1}\right), x_{2}-p_{2}\left(x_{2}\right), x_{3}-p_{3}\left(x_{3}\right)\right)\right. \\
\left.-\phi_{i} \circ N_{i}\left(u_{i 1}, u_{i 2}, u_{i 3}\right)\right], \quad i=1,2,3 .
\end{gathered}
$$

By Lemma $4.1,\left(x_{1}, x_{2}, x_{3}, u_{11}, u_{12}, u_{13}, u_{21}, u_{22}, u_{23}, u_{31}, u_{32}, u_{33}\right)$ is a solution of the SGVIP (4.1). This completes the proof.

\section{References}

[1] R. P. Agarwal, Y. J. Cho, and N. J. Huang, "Sensitivity analysis for strongly nonlinear quasi-variational inclusions," Applied Mathematics Letters, vol. 13, no. 6, pp. 19-24, 2000.

[2] R. P. Agarwal, N. J. Huang, and Y. J. Cho, "Generalized nonlinear mixed implicit quasi-variational inclusions with set-valued mappings," Journal of Inequalities and Applications, vol. 7, no. 6, pp. 807$828,2002$.

[3] R. Ahmad and Q. H. Ansari, "An iterative algorithm for generalized nonlinear variational inclusions," Applied Mathematics Letters, vol. 13, no. 5, pp. 23-26, 2000.

[4] S.-S. Chang, Y. J. Cho, and H. Y. Zho, "On the iterative approximation methods of fixed points for asymptotically contractive type mappings in Banach spaces," in Fixed Point Theory and Applications. Vol. 3, pp. 33-41, Nova Science Publishers, Huntington, NY, USA, 2002.

[5] X. P. Ding, "Existence and algorithm of solutions for generalized mixed implicit quasi-variational inequalities," Applied Mathematics and Computation, vol. 113, no. 1, pp. 67-80, 2000.

[6] N.-J. Huang and Y.-P. Fang, "A new class of general variational inclusions involving maximal $\eta$ monotone mappings," Publicationes Mathematicae Debrecen, vol. 62, no. 1-2, pp. 83-98, 2003. 
[7] R. U. Verma, "A-monotonicity and applications to nonlinear variational inclusion problems," Journal of Applied Mathematics and Stochastic Analysis, vol. 17, no. 2, pp. 193-195, 2004.

[8] Y. P. Fang and N. J. Huang, "Approximate solutions for nonlinear operator inclusions with $-(H, \eta)$ monotone operators," Tech. Rep., Sichuan University, 2003.

[9] Y.-P. Fang and N.-J. Huang, "H-accretive operators and resolvent operator technique for solving variational inclusions in Banach spaces," Applied Mathematics Letters, vol. 17, no. 6, pp. 647-653, 2004.

[10] N. J. Huang and Y. P. Fang, "Generalized m-accretive mappings in Banach spaces," Journal of Sichuan University, vol. 38, no. 4, pp. 591-592, 2001.

[11] Y. P. Fang, Y. J. Cho, and J. K. Kim, “ $(H, \eta)$-accretive operators and approximating solutions for systems of variational inclusions in Banach spaces," Nonlinear Analysis: Theory, Methods $\mathcal{E}$ Applications. In press.

[12] K. R. Kazmi and F. A. Khan, "Iterative approximation of a solution of multi-valued variational-like inclusion in Banach spaces: a P- $\eta$-proximal-point mapping approach," Journal of Mathematical Analysis and Applications, vol. 325, no. 1, pp. 665-674, 2007.

[13] H.-Y. Lan, “On multivalued nonlinear variational inclusion problems with $(A, \eta)$-accretive mappings in Banach spaces," Journal of Inequalities and Applications, Article ID 59836, 12 pages, 2006.

[14] H.-Y. Lan, Y. J. Cho, and R. U. Verma, "Nonlinear relaxed cocoercive variational inclusions involving $(A, \eta)$-accretive mappings in Banach spaces," Computers $\mathcal{E}$ Mathematics with Applications, vol. 51, no. 9-10, pp. 1529-1538, 2006.

[15] X.-P. Luo and N.-J. Huang, " $(H, \eta)-\eta$-monotone operators in Banach spaces with an application to variational inclusions," Applied Mathematics and Computation, vol. 216, no. 4, pp. 1131-1139, 2010.

[16] H. K. Xu, "Inequalities in Banach spaces with applications," Nonlinear Analysis: Theory, Methods $\mathcal{E}$ Applications, vol. 16, no. 12, pp. 1127-1138, 1991.

[17] Z. B. Xu and G. F. Roach, "Characteristic inequalities of uniformly convex and uniformly smooth Banach spaces," Journal of Mathematical Analysis and Applications, vol. 157, no. 1, pp. 189-210, 1991.

[18] I. Cioranescu, Geometry of Banach Spaces, Duality Mappings and Nonlinear Problems, vol. 62 of Mathematics and Its Applications, Kluwer Academic Publishers, Dordrecht, The Netherlands, 1990.

[19] H. K. Xu, "Inequalities in Banach spaces with applications," Nonlinear Analysis: Theory, Methods $\mathcal{E}$ Applications, vol. 16, no. 12, pp. 1127-1138, 1991.

[20] H.-Y. Lan, "Stability of iterative processes with errors for a system of nonlinear $(A, \eta)$-accretive variational inclusions in Banach spaces," Computers \& Mathematics with Applications, vol. 56, no. 1, pp. 290-303, 2008.

[21] E. Zeidler, Functional Analysis and its Applications II: Monotone Operators, Springer, New York, NY, USA, 1985.

[22] Y. J. Cho and X. Qin, "Systems of generalized nonlinear variational inequalities and its projection methods," Nonlinear Analysis: Theory, Methods E Applications, vol. 69, no. 12, pp. 4443-4451, 2008.

[23] Y. J. Cho, Y. P. Fang, N. J. Huang, and H. J. Hwang, "Algorithms for systems of nonlinear variational inequalities," Journal of the Korean Mathematical Society, vol. 41, no. 3, pp. 489-499, 2004.

[24] G. Kassay and J. Kolumbán, "System of multi-valued variational inequalities," Publicationes Mathematicae Debrecen, vol. 54, pp. 267-279, 1999.

[25] R. U. Verma, "Generalized system for relaxed cocoercive variational inequalities and projection methods," Journal of Optimization Theory and Applications, vol. 121, no. 1, pp. 203-210, 2004.

[26] S. B. Nodler, "Multivalued contraction mapping," Pacific Journal of Mathematics, vol. 30, no. 3, pp. 457-488, 1969. 


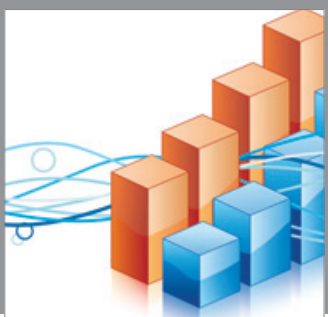

Advances in

Operations Research

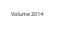

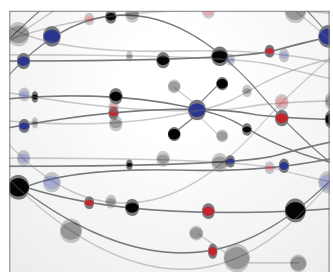

\section{The Scientific} World Journal
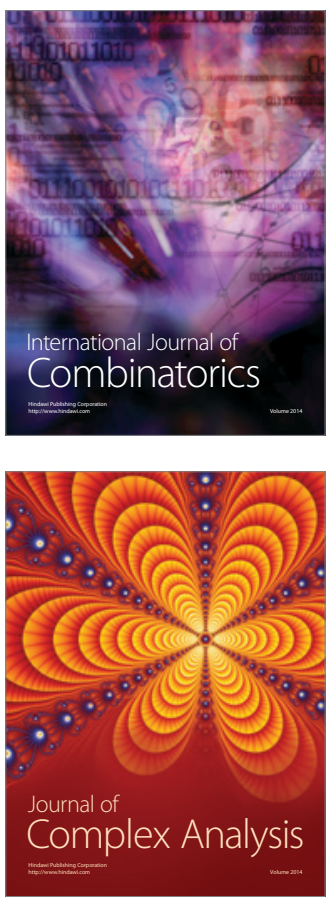

International Journal of

Mathematics and

Mathematical

Sciences
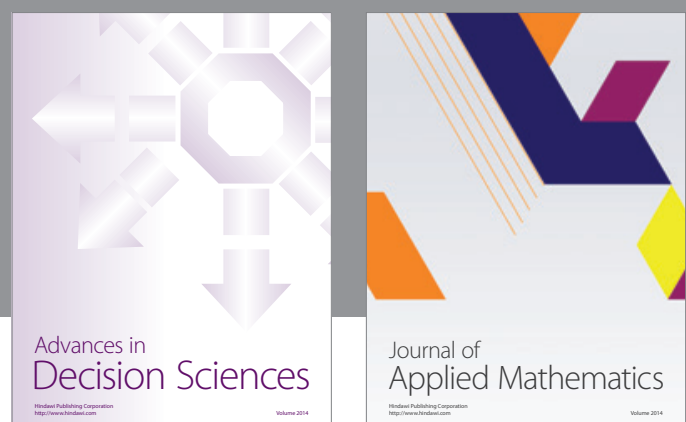

Journal of

Applied Mathematics
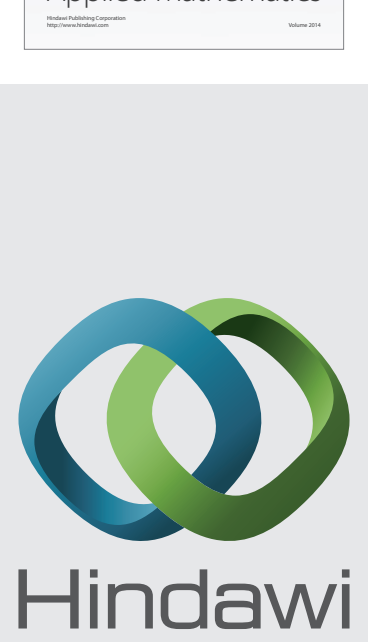

Submit your manuscripts at http://www.hindawi.com
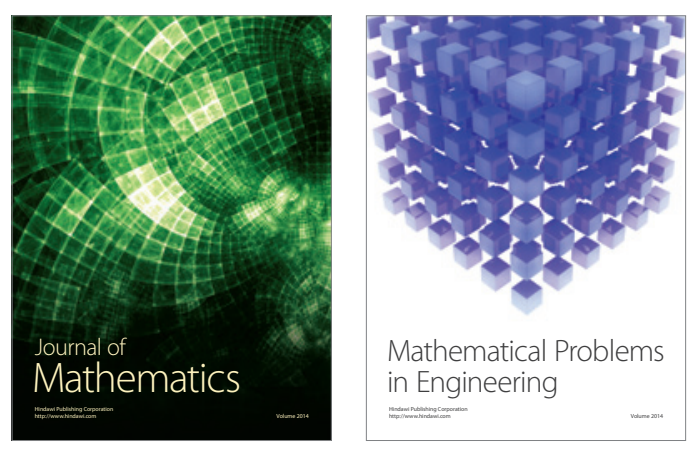

Mathematical Problems in Engineering
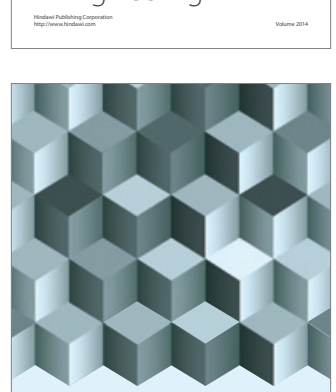

Journal of

Function Spaces
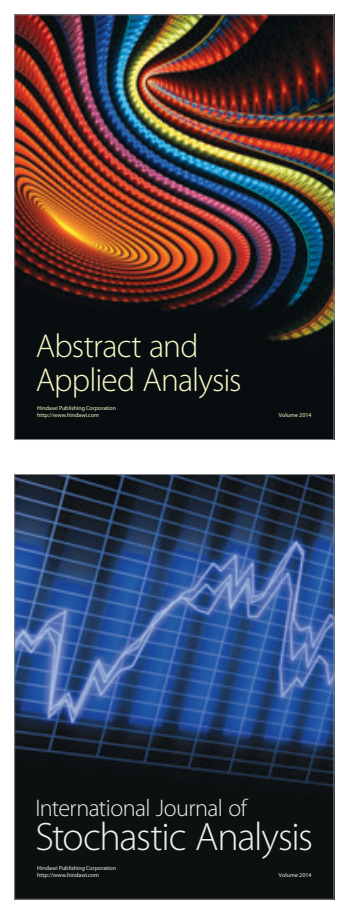

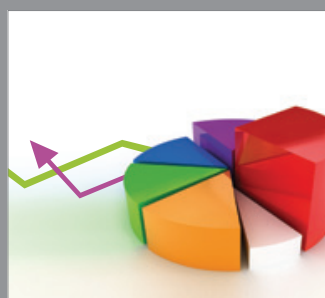

ournal of

Probability and Statistics

Promensencen
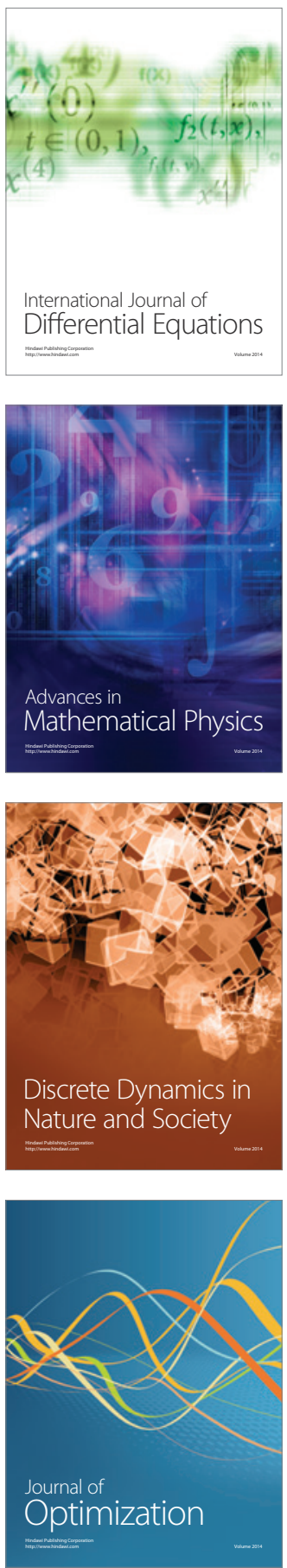